\title{
WILEY-VCH
}

\section{Surface modification of (non)-fluorinated vitrimers through dynamic transamination}

Christian Taplan, Marc Guerre, Christopher N. Bowman*, and Filip E. Du Prez*

C. Taplan, Dr. M. Guerre, Prof. F. E. Du Prez

Polymer Chemistry Research Group, Centre of Macromolecular Chemistry (CMaC), Department of Organic and Macromolecular Chemistry, Faculty of Sciences, Ghent University, Krijgslaan 281 S4-bis, Ghent B-9000, Belgium

E-mail: filip.duprez@ugent.be

Dr. M. Guerre

Laboratoire des IMRCP, Université de Toulouse, CNRS UMR5623, Université Paul Sabatier, 118 route de Narbonne, 31062 Toulouse Cedex 9, France

Prof. C. N. Bowman

Department of Chemical and Biological Engineering, University of Colorado, Campus Box 596, Boulder, Colorado 80309-0596, United States

E-mail: christopher.bowman@colorado.edu

Keywords: covalent adaptable networks, vitrimers, nano-imprint lithography, vinylogous urethane

\begin{abstract}
Surface modifications are typically permanent in shape and chemistry. Herein, vinylogous urethane (VU) chemistry is presented as an easily accessible and versatile platform for rapid, facile and reworkable surface modification. It is demonstrated that both physical and chemical post-modification of permanent, yet dynamic elastic polymer networks are achieved. Surface patterns with high regularity are created, both via a straightforward replication process using a PDMS stamp (resolution ca. 10-100 $\mu \mathrm{m}$ ) as well as using thermally activated nano-imprint lithography (NIL) to form hole, pillar or line patterns (ca. $300 \mathrm{~nm}$ ) in elastic VU-based vitrimers. The tunable, rapid exchange allows patterning at $130^{\circ} \mathrm{C}$ in less than $15 \mathrm{~min}$, resulting in an increased water contact angle and surface-structure induced light reflection. Moreover, it is also demonstrated that the use of a single dynamic covalent chemistry makes it possible to strongly adhere fluorinated and non-fluorinated materials based on incompatible matrices, causing cohesive failure in a peel test. In a topography scan, the visibly transparent interface is
\end{abstract}




\section{WILEY-VCH}

shown to possess a continuous phase without a gap, while maintaining distinctively separated (non)fluorinated domains. Finally, this approach allowed for a straightforward coating of a nonfluorinated material with a fluorinated monomer to minimize the overall fluorinated content.

\section{Introduction}

In the past decades, inspired by the perspective that all plastic and polymeric materials will eventually need to be reprocessable and fully recyclable, attention has turned towards solutions for otherwise unrecyclable polymeric materials (i.e., thermosets). ${ }^{[1]}$ These materials are threedimensional, permanently crosslinked covalent polymer networks that are used for demanding applications, often requiring both mechanical and chemical resistance. Thermosetting materials are not only used as bulk materials, but they are also widely applied as for instance protective coatings or to introduce a desired surface functionality in various applications. Indeed, various types of surface modification and structuration are readily used in thermosetting materials, in which often a liquid resin or solution is cured by exposure to a certain stimulus such as for instance light or heat in a patterned mold over different length scales. ${ }^{[2]}$ The resulting surface then possesses specific surface properties such as super-hydrophobicity. However, this process also suffers from an inherent irreversibility and often inherent stress that results from the curing process. Moreover, these surface morphologies possess a permanently crosslinked structure that makes it difficult to further post-modify, reshape, or repair them. In this context, the implementation of functional reactive crosslinks that are able to (re)form and dissociate or rearrange reversibly to overcome the inherent non-recyclability of covalently crosslinked polymer networks is an interesting strategy. This concept, commonly referred to as covalent adaptable networks (CANs), ${ }^{[3]}$ has been explored for a number of different crosslinked materials and considered for broad applications ranging from polymer processing, ${ }^{[4]}$ creation of composites, ${ }^{[5]}$ welding, ${ }^{[6]}$ or adhering ${ }^{[4 a,}{ }^{7]}$ to structuration, ${ }^{[8]}$ enabling significant technological advancements that address societal interests in sustainable materials. Vitrimers, 


\section{WILEY-VCH}

pioneered by Leibler and co-workers in 2011, are a unique and promising class among these covalent adaptable networks. ${ }^{[9]}$ They are permanently cross-linked polymeric materials that exhibit visco-elastic liquid-like behavior upon heating wherein the macroscopic deformation is controlled by the rate of the dynamic covalent exchange reactions. ${ }^{[10]}$ At elevated temperatures, dynamic bond rearrangement promotes large scale macroscopic flow, thus enabling reversible deformation, weldability, reprocessing, and recycling in a similar way to vitreous silica (glass). Moreover, in contrast to dissociative reversible networks, associative vitrimeric networks keep their network integrity throughout the reorganization procedure. ${ }^{[1]}$ Recently, various dynamic covalent chemistry platforms were proposed based on among others transesterification, ${ }^{[9,12]}$ silyl-ether exchange, ${ }^{[13]}$ disulfide exchange, ${ }^{[14]}$ trans-thioesterification, ${ }^{[15]}$ trans-alkylation, ${ }^{[16]}$ dioxaborolane metathesis, ${ }^{[4 a]}$ vinylogous urethane/urea transamination, ${ }^{[17]}$ and others. ${ }^{[18]}$ Several of those platforms have also been explored for demonstration of surface patterning, assembling or welding of composites, and adhering different crosslinked parts. ${ }^{[8 a, 19]}$ However, most of these reactions take place with compatible or similar materials with a glass transition temperature $\left(T_{g}\right)$ above room temperature, and processing either over a period of hours or requiring very high temperatures. Additionally, while transesterification is arguably among the most commonly applied exchange platform, it was shown that vinylogous urethane transamination is an easily accessible, yet versatile exchange chemistry platform for the creation of dynamic networks. ${ }^{[17 b, 20]}$ Indeed, vinylogous urethane transamination allows for swift exchange rates, both catalyzed and non-catalyzed that are able to be implemented in a broad range of different matrices, covering a range from glassy polymers up to (non)fluorinated elastomers. ${ }^{[20-21]}$

In this work, a rapid, straightforward post-synthetic physical and chemical surface modification of permanently crosslinked networks is presented, which is demonstrated to work with both catalyzed and non-catalyzed (non)-fluorinated vinylogous urethane vitrimers (Figure 


\section{WILEY-VCH}

1). Physical modification-based imprinting of surface structures is demonstrated on micron and submicron-scales by using a patterned polydimethylsiloxane (PDMS) stamp, as it is commonly used in lithographic structuration, and thermally activated nano-imprint lithography (NIL), respectively. In addition to exploring different patterns, imprinted fluorinated materials are compared to their non-fluorinated counterparts with regard to their temporal stability and surface properties. A fluorinated vitrimer is expected to possess a lower surface energy as their non-fluorinated counterparts. The latter ones are used as an elastic reference material and the achieved tunability of their dynamic exchange rate is transferred to fluorinated vinylogous urethane vitrimers. Chemical surface modification is exemplified via lamination and coating of otherwise incompatible, fluorinated materials based on a perfluorinated polyether (PFPE) with non-fluorinated resins based on polypropylene glycol (PPG), leveraging the provided surface functionalities and inherently swift reactivity.

a)

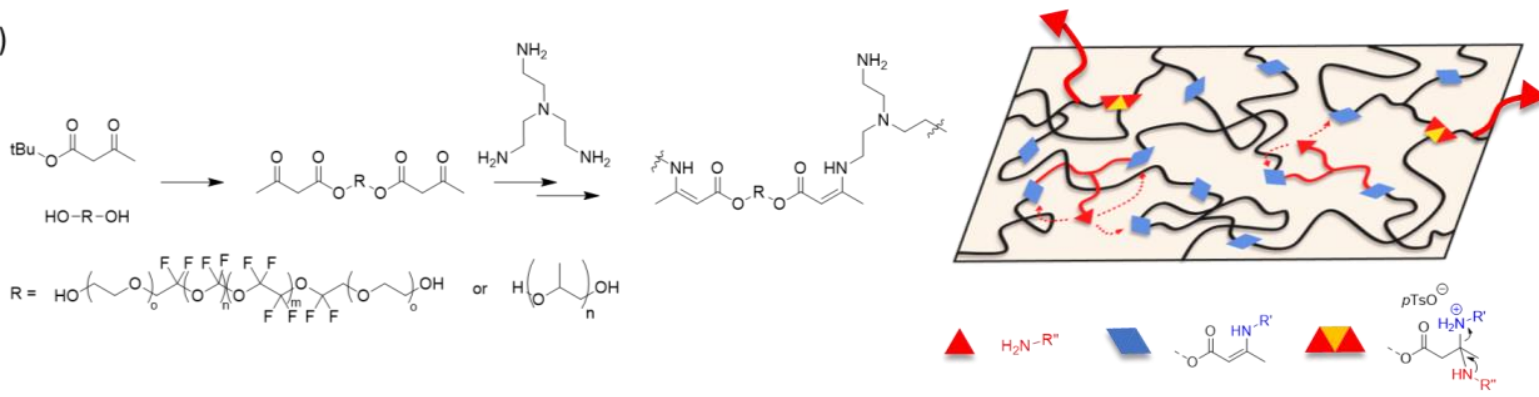

b)
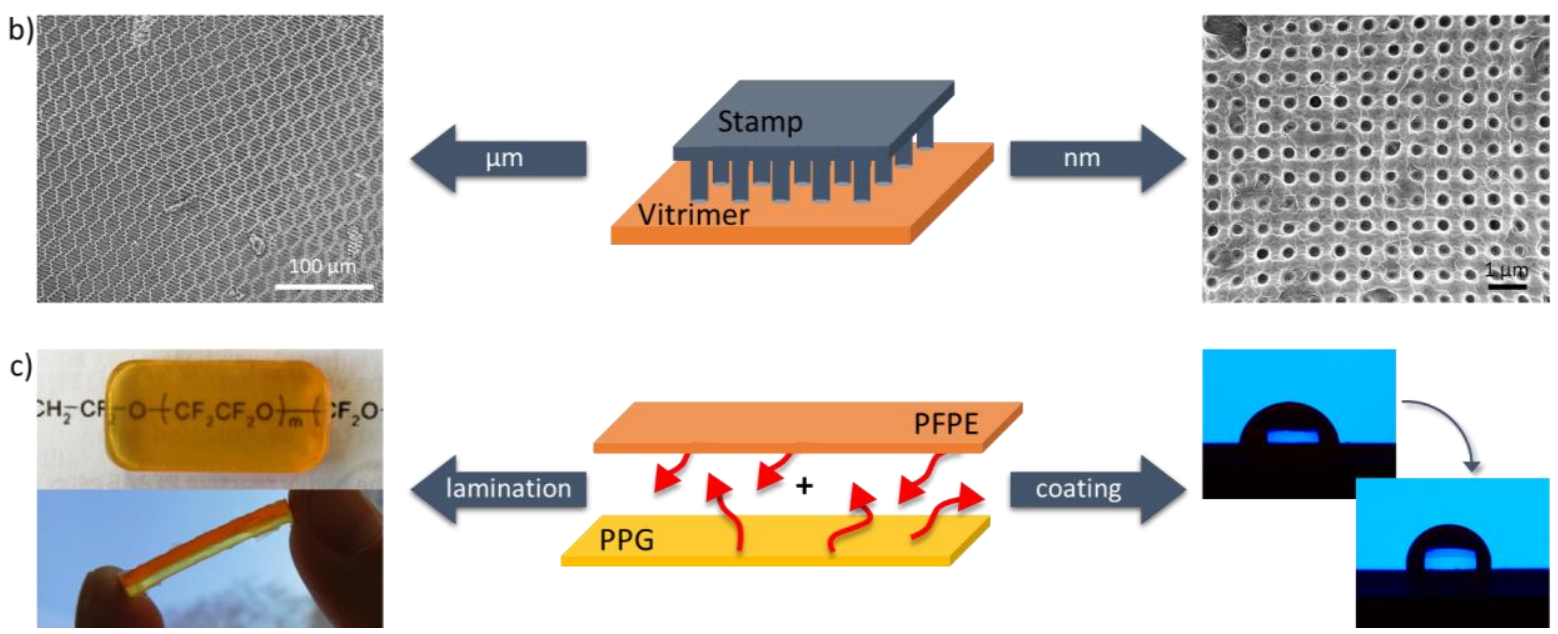

Figure 1. Schematic overview of the monomer and network synthesis and reaction mechanism of the transamination of the vinylogous urethane moiety (a), schematic representation of 


\section{WILEY-VCH}

physical surface patterning (b) using a PDMS stamp for micropatterning (left) and thermally activated nano-imprinting lithography (right), and (c) chemical modification via lamination of different vitrimers (left) or coating with a fluorinated building block (right).

\section{Results and Discussion}

\subsection{Vitrimer synthesis and characterization}

The necessary monomers to prepare the investigated vitrimers were synthesized via acetoacetylation of the fluorinated and non-fluorinated polyether diols. This was accomplished in a straightforward solvent-free one-pot vacuum distillation, in which tert-butyl alcohol as a small molecule reaction product was removed successively throughout the reaction, followed by removal of unreacted tert-butyl acetoacetate. The obtained monomers were combined with tris(2-aminoethyl)amine (TREN) as trifunctional nucleophilic crosslinker ( $\mathrm{A}_{3}$-type) in accordance to earlier reports to yield vitrimers with 5 or 20 mol.\% pendent primary amines (Figure 1a) and is referred to as ' $\mathrm{N} 5$ ' or ' $\mathrm{N} 20$ '..$^{[4 \mathrm{~b}, 21 \mathrm{~d}]}$ Completion of the network curing was confirmed via ATR-FTIR spectroscopy (Figure S1a). To further provide materials capable of rearranging sufficiently swiftly, meaning to release the applied stress in the range of seconds to minutes (at elevated temperatures), an earlier strategy was followed including the addition of 6 mol.\% para-toluene sulfonic acid ( $p \mathrm{TsOH}$ ) as a protic catalyst (referred to as ' $\mathrm{H} 6$ '). It was previously found that the addition of this Brønsted acid enhances the kinetics of the dynamic covalent bond exchange by suppressing a dual-temperature responsive behavior in the temperature window ranging from $90^{\circ} \mathrm{C}-160^{\circ} \mathrm{C} \cdot{ }^{[4 \mathrm{~b}, 17 \mathrm{a}]}$ To verify this feature for the herein investigated PFPE-based fluorinated vitrimers, stress relaxation experiments were performed (Figure S1b), resulting in a single activation energy as indicated by a linear Arrhenius-plot (Figure S2). 


\section{WILEY-VCH}

The PFPE-based materials possess two glass transition temperatures ( $\mathrm{Tg}$ ), respectively linked to the $\mathrm{Tg}$ of the fluorinated domain (around $-100^{\circ} \mathrm{C}$ ) and the non-fluorinated, vinylogous urethane part (around $-40^{\circ} \mathrm{C}$ ), as determined by DSC measurements (Figure S3). A nonfluorinated polypropylene glycol (PPG) reference material, also containing 5 mol.\% pendent primary amines, was reported to possess a $T_{g}$ of $-10^{\circ} \mathrm{C} \cdot{ }^{[4 b]}$ The thermal degradation onset temperature at $5 \mathrm{wt} . \%$ weight loss was determined to be $240{ }^{\circ} \mathrm{C}$ for the catalyzed materials (PFPE-N5H6 and PFPE N20H6) and $280{ }^{\circ} \mathrm{C}$ for the non-catalyzed PFPE-N5 (Figure S4). This showed that each of the created materials was elastic during handling and processing, as well as thermally stable throughout its handling and imprinting. Due to their soft elastic behavior and their inherent ability to dissipate externally applied stress swiftly, even at relatively low temperatures, it was hypothesized that these materials would easily and rapidly replicate a (sub)-microstructural pattern when exposed to heat and a patterned stamp or a structured mold.

\subsection{Micro-patterning of vitrimers}

To demonstrate the possibility of micro-structural modification, a simple set-up was considered consisting of a Sharklet ${ }^{\circledR}$-patterned PDMS-stamp that was placed on top of a PFPE N5H6 vitrimer film with both parts sandwiched between two glass slides to evenly distribute the applied forces (Figure 2). As directive force, a $1.5 \mathrm{~kg}$ weight was placed on the top glass slide, placed in an oven at $120^{\circ} \mathrm{C}$ and left for $15 \mathrm{~min}$ for a thin film (thickness approximately $500 \mu \mathrm{m}$ ), and $2 \mathrm{~h}$ in case of a $2 \mathrm{~mm}$ thick vitrimer sheet to make sure the material was sufficiently heated to dissipate the applied stress (Figure S5). Interestingly, with this basic setup the material was able to become micro-structured, as seen visually from surface-structure induced light reflection (Figure 2 a), bottom).

When assessing the structures with an optical and an electron microscope, it was observed that the obtained pattern showed a very high degree of regularity across different length-scales down to microns. This demonstrates the facile possibility to imprint a defined pattern into a soft 


\section{WILEY-VCH}

permanent network, and opens a variety of opportunities, for instance to replicate structures of PDMS stamps and afterwards easily recreate the original via for instance light-induced polymerization of a photosensitive solution.

a)

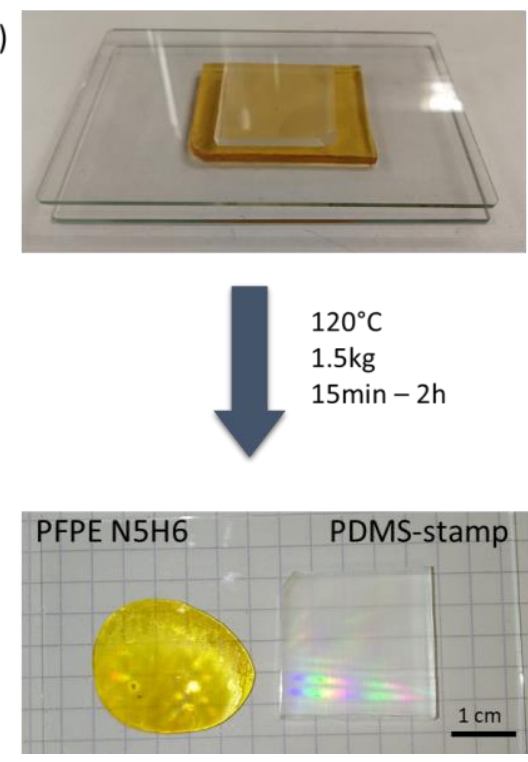

b)

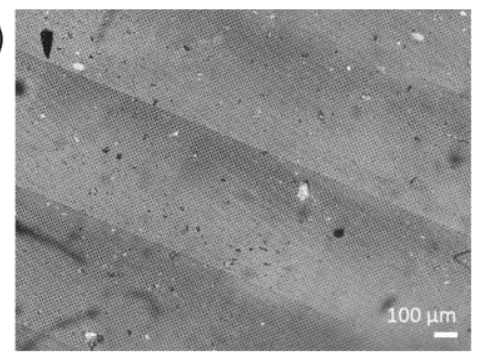

c)

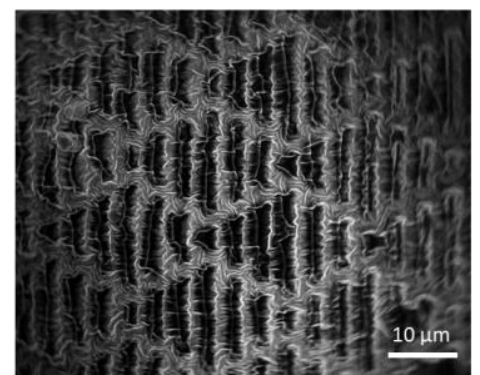

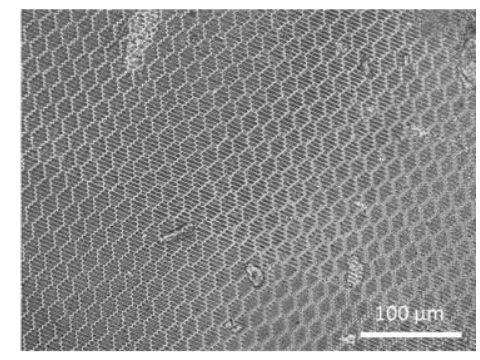

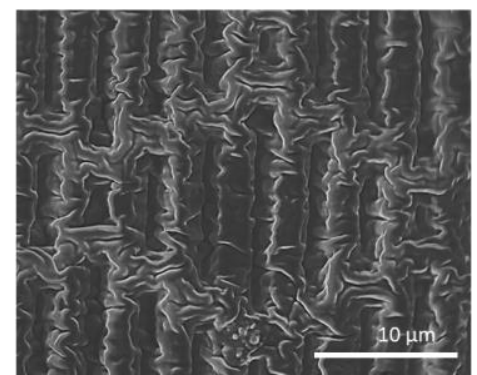

Figure 2. Imprinting of micro-structures into a fluorinated vitrimer with a Sharklet ${ }^{\circledR}$-patterned PDMS stamp (a), and its regularly replicated structure on vitrimer PFPE N5H6 observed via optical microscopy (b) and scanning electron microscopy (c).

This approach is possible since PFPE N5 was shown to have a low swelling degree in various non-fluorinated organic solvents (e.g. 8, 20 and $35 \%$ in methanol, DMF and THF respectively). ${ }^{[21 \mathrm{~d}]}$ Hence, imprinting a fluorinated vitrimer could be advantageous because thanks to the fluorinated matrix - swelling is limited, and network integrity is kept because of the associative nature of the exchange. This behavior is likely to facilitate reproduction of the pattern, while providing a reprint-able and reusable stamp. This was indicated when the created pattern was erased and reprinted following the same procedure (Figure S6), while withstanding for $1 \mathrm{~h}$ at $120^{\circ} \mathrm{C}$ without pressure. In fact, the associative nature of the dynamic covalent exchange allows to maintain network integrity, preventing from immediate structural collapse. 


\section{WILEY-VCH}

\subsection{Thermally activated nano-imprint lithography of VU vitrimers}

To show further the versatility of imprinting fluorinated elastic substrates, nano-imprint lithography was considered. This technique was earlier used to create sub-micron patterns by applying an external stimulus such as heat, light, pressure or combinations thereof onto a substrate able to fill a patterned mold. Typically, patterns were created via UV-curing of liquid monomers in a mold, or by making use of shape-memory materials exposed to pressure and heat. ${ }^{[2 \mathrm{a}]}$ In a few recent cases, dynamic networks were explored, such as liquid crystalline networks, CANs and vitrimers, all of which made use of a $T_{m}$, or $T_{g}$ beyond room temperature,

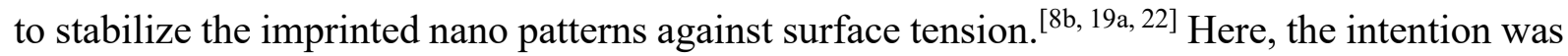
to determine whether sub-micron patterns could be created in elastic networks and how long they would last, considering the possibility to rearrange the network topology at lower temperatures, and also to compare this behavior to non-fluorinated materials.

In order to investigate if a submicron pattern could be created and noticeably retrieved afterwards, the PFPE N5H6 material was compared to a permanently crosslinked polyurethane elastomer reference. Both networks were exposed to a pillar patterned silicon mold at $130{ }^{\circ} \mathrm{C}$ with 25 bar air pressure. The applied pressure was in the mid-range of the possibly applied pressure and proved to be sufficient as a directive force for these dynamic networks, placing the material under stress to flow around or inside the mold. The time-temperature conditions upon which imprinting was performed were based on the thermal stability assessment and stress relaxation experiments. At $130{ }^{\circ} \mathrm{C}$, the material of choice (PFPE N5H6) dissipated more than $99 \%$ of the initially applied shear stress within 5 min (Figure S1-2). Additionally, this temperature was far below the degradation temperature (Figure S4). Hence, the applied temperature was safe to use and considered to enable sufficiently fast conditions for thermally activated nano-imprinting. On the other hand, the polyurethane network was exposed to three times the imprinting time (i.e. $15 \mathrm{~min}$ ) to assess possible effects originating from, e.g., hydrogen bond formation. Imprinting via NIL of this permanent network did not result in a retrievable 


\section{WILEY-VCH}

pattern (Figure S7). In contrast to the PFPE N5H6 vitrimer, this excludes possible shapememory effects related to hydrogen bonding responsible for the obtained patterns in the dynamic networks. Upon removal of the Si-mold from the dynamic network, light reflections were observed from the imprinted region of the material, indicating that the desired repeating (sub)micron pattern had been created. Indeed, when looking at the imprinted material using SEM, the desired patterns were observed with good fidelity to the original process (Figure 3).
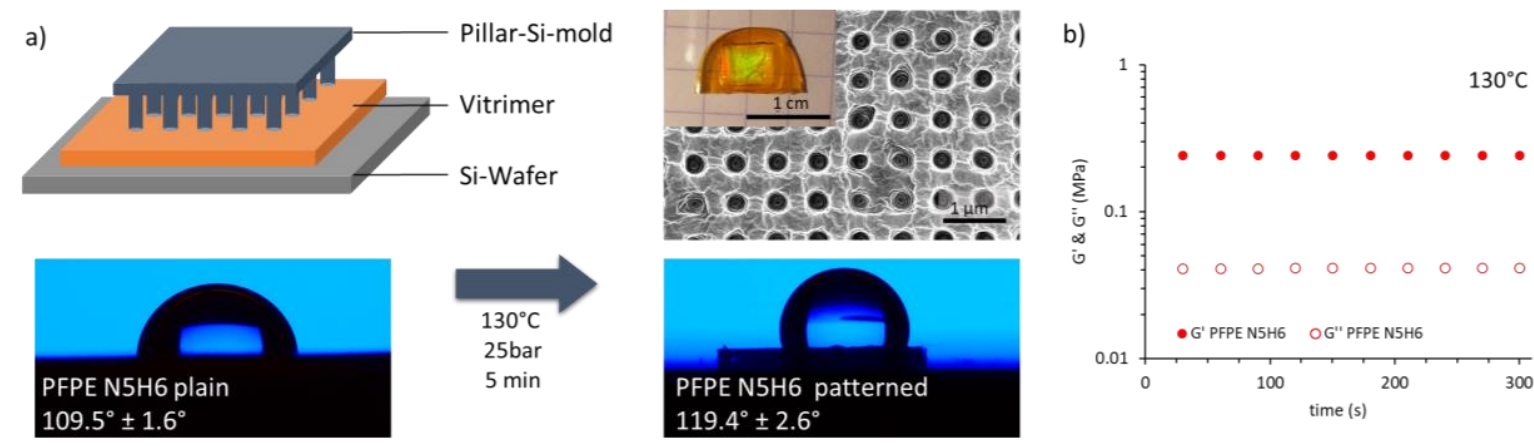

Figure 3. Schematic display of the nano-imprint lithography of submicron patterns on PFPE N5H6 and change of the contact angle (a) and a time-sweep showing a constant shear storage and loss modulus during the imprinting time (b).

\subsection{Structural versatility and temporal stability}

Next, imprinting was performed exploring different patterns in combination with variously patterned Si-molds (pillars, holes and lines) for nano-imprinting (Figure S8a). The investigated pillar and hole patterned molds show a diameter of around $300 \mathrm{~nm}$ for both pillars and holes, determined via AFM (Figure S9) and confirmed with regard to their observed diameters via SEM (Figure S8c). The depth of the patterns varied from 150-250 nm for pillars, and 70-80 nm for holes. The line patterned mold showed a width of 250-300 nm with a distance between lines of around 350-400 $\mathrm{nm}$ as determined from the SEM image. The effect of the structuration was exemplified by contact angle measurements and the resulting change of the material contact 


\section{WILEY-VCH}

angle when comparing a non-patterned vitrimer film with a patterned vitrimer material (Figure

\section{3a, Figure S10).}

The potential of vinylogous urethane based vitrimers for straightforward and rapid nanoimprinting, different elastic (non-)fluorinated substrates have also been considered in this study. Since each pattern was able to result in a successfully imprinted negative (Figure 4a), one mold was selected to explore other substrates. Therefore, a pillar patterned Si-mold was selected to imprint holes onto various substrates. The imprinting time in this context was adjusted taking into account the presence (or absence) of a catalyst, ranging from $900 \mathrm{~s}$, similar to the irreversibly crosslinked reference, for non-catalyzed PFPE N5 and PPG N5, to $300 \mathrm{~s}$ and $150 \mathrm{~s}$ for catalyzed PFPE N5H6 and PFPE N20H6, respectively (Figure 4b).

a)

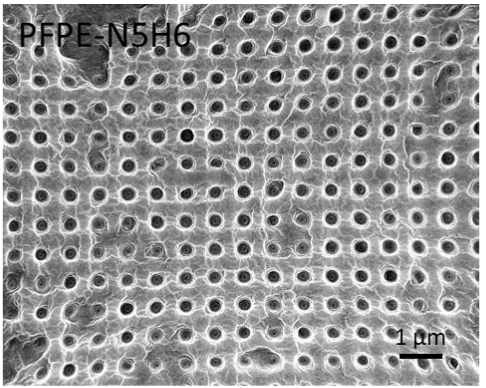

b)

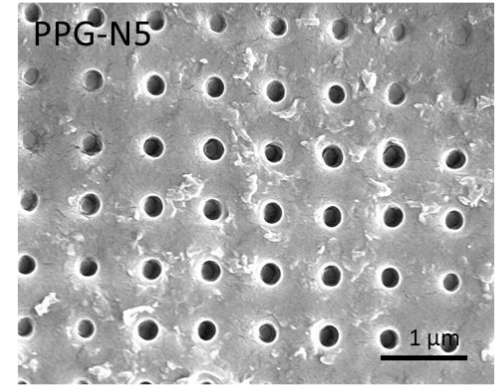

c)

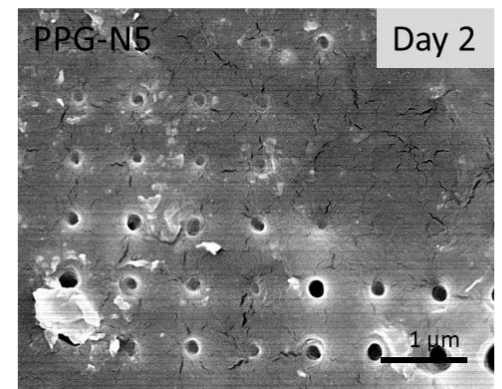

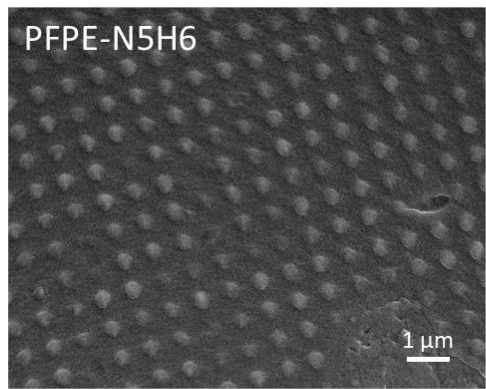
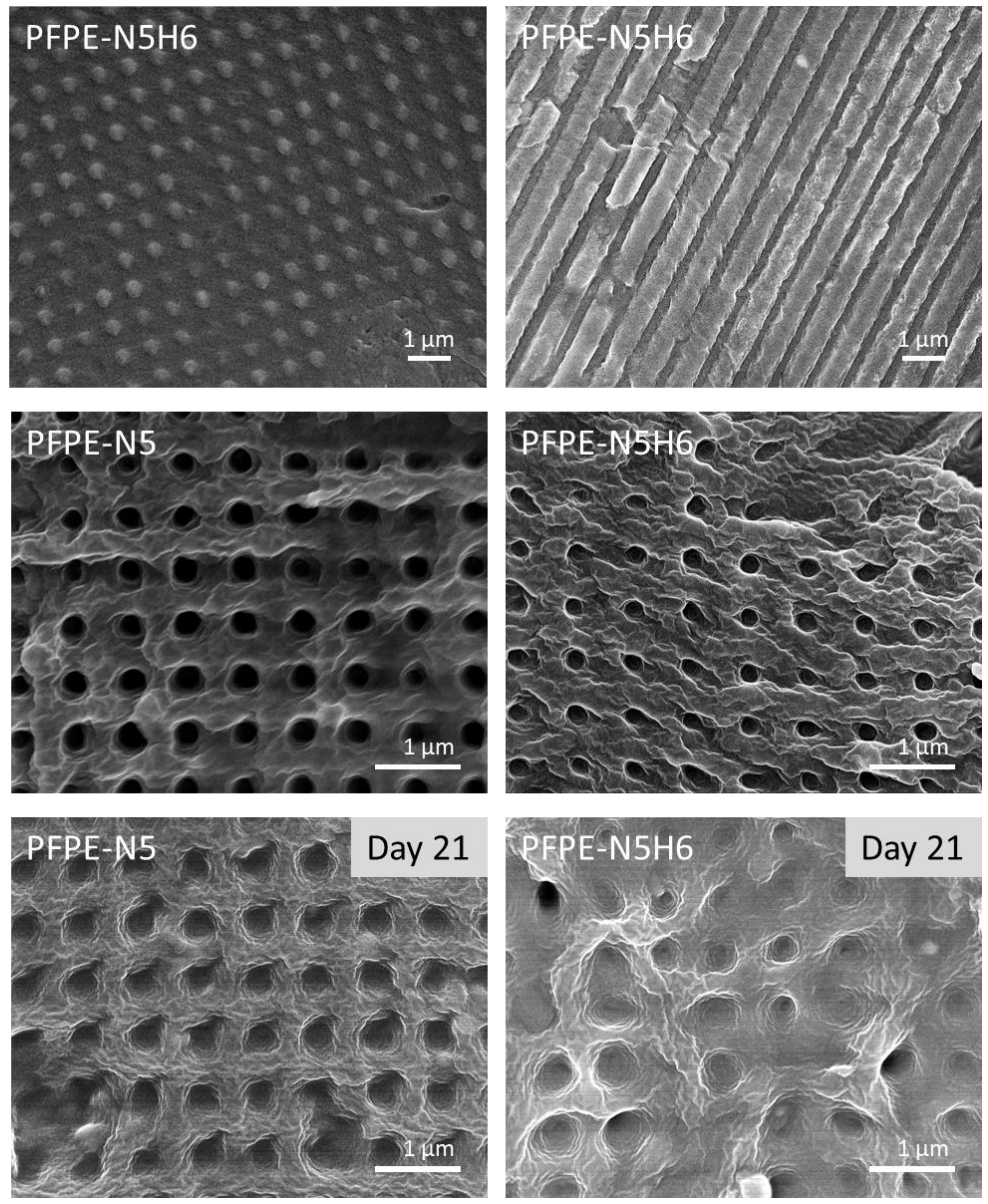


\section{WILEY-VCH}

Figure 4. SEM images of replicated patterns such as hole, pillar and line patterned PFPE N5H6 showing structural versatility (a), as well as different imprinted materials (b) and their respective temporal changes after two days for PPG- and 21 days for PFPE-based vitrimers (c).

It should be noted that PFPE N20H6 was prepared for imprinting in a manner similar to the other materials. However, it did not result in a retrievable pattern, possibly because of the accelerated relaxation linked to the higher content of primary amine moieties and pTsOH. In contrast, dynamically crosslinked materials with a lower exchange rate displayed well retrievable sub-micron hole patterns with a hole diameter derived via SEM images of around $300 \mathrm{~nm}$ (Figure $4 \mathrm{~b}$ and Figure S8c). When monitoring the obtained patterns over time, a clear difference became apparent between the temporal stability of patterned fluorinated and patterned non-fluorinated materials, which is believed to be related to a lower surface energy, expressed by the fluorinated parts of the polymer networks, reducing the driving force of minimizing surface energy compared to non-fluorinated materials. Indeed, despite the fact that the non-fluorinated PPG N5 possesses a lower exchange rate than the catalyzed PFPE N5H6, it is observed that the pattern already becomes significantly weaker after two days, whereas on fluorinated materials the patterns, although also attenuated, are still detectable after 21 days (Figure 4c) and might be useful, among others, in the creation of surfaces with transient surface properties.

\subsection{Lamination of (non)-fluorinated vitrimers}

Imprinting induced patterning, however, was not the only straightforward approach that was applied to a dynamic network comprising active functional and reactive moieties in order to modify a material's surface. Dynamic covalent chemistries also provided the possibility for welding or adhering materials with similar matrices and could, thanks to the reversible reactions involved, also form strong binding interactions. In fact, this is the underlying principle when a ground material is reprocessed, e.g. via compression molding. At the interfaces, where the 


\section{WILEY-VCH}

materials are brought in contact, they start to react by means of their reversible exchange reactions, and in the case of compatible matrices, they are successfully healed into new, larger material plates or films. This behavior was expected to be more difficult when using incompatible matrices, because separated domains are likely to form. However, when the main domains were kept separate and only brought in contact on a limited area at the interface (Figure S11), strong adhesion was apparent. For example, when performing a peel test, cohesive failure was observed (video provided in the supporting information). Moreover, when compressing two intact polymer layers with different polymer matrices (fluorinated and non-fluorinated) via compression molding, aside from the fact that the layers could not be peeled off each other without harming the other layer, a transparent interface was obtained (Figure 5a).

a)
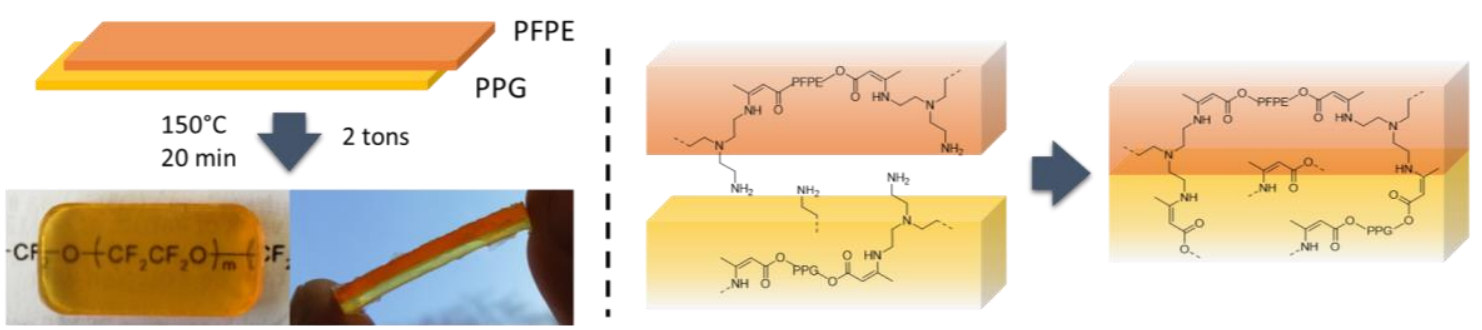

b)
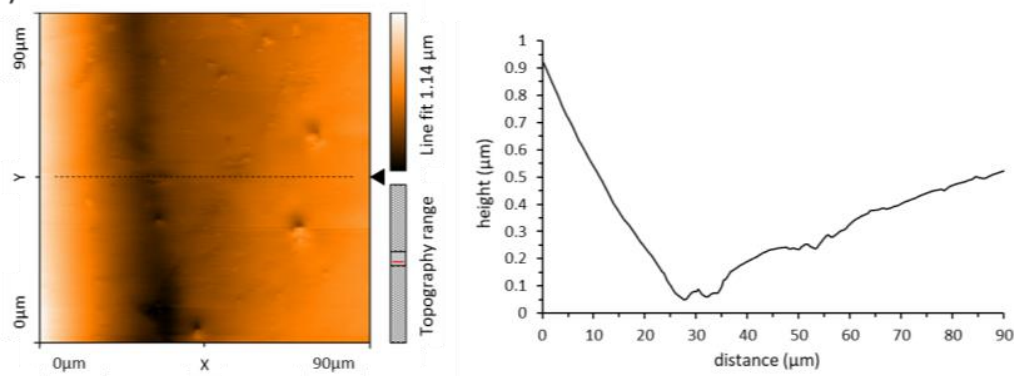

c)

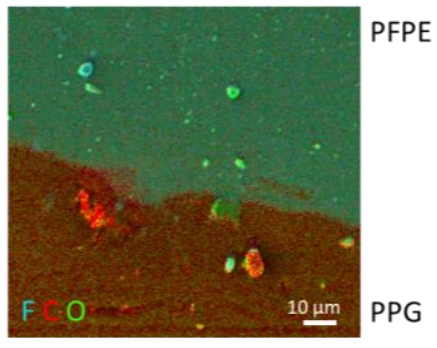

Figure 5. Schematization of the lamination of non-fluorinated PPG- and fluorinated PFPEbased vinylogous urethane vitrimers (a), AFM topography scan and a height profile (b) EDS overlay image over the elemental distribution of $\mathrm{F}$ (cyan), $\mathrm{C}$ (red) and $\mathrm{O}$ (green) (c).

Performing a topography scan on a cross-section of this interface did not show a gap but rather a continuous phase of the two materials across the interface. In addition, via energy- 


\section{WILEY-VCH}

dispersive x-ray spectroscopy (EDS), both for catalyzed and non-catalyzed material interfaces, a clear separation of the fluorinated and non-fluorinated section of the material was observed (Figure 5c, Figure S12-13). This boundary was also observed via the elemental distribution obtained after a line-scan was performed.

\subsection{Functional coatings}

These insights confirmed the hypothesis that functional groups at the surface of the vinylogous urethane vitrimers were reacting swiftly and efficiently with compatible reactive functional moieties, despite the fact that the bearing polymers themselves might not be compatible or miscible. This behavior enables a straightforward surface modification with fluorinated materials. To demonstrate this, a non-fluorinated PPG N5 vitrimer was covered with a drop of PFPE-acetoacetate (PFPE-AA), placed in an oven and heated at $120{ }^{\circ} \mathrm{C}$ for $10 \mathrm{~min}$ to assure that small amounts of water, formed from the condensation reaction, could evaporate and to drastically alter the surface properties, as exemplified with a change in contact angle (Figure 6).
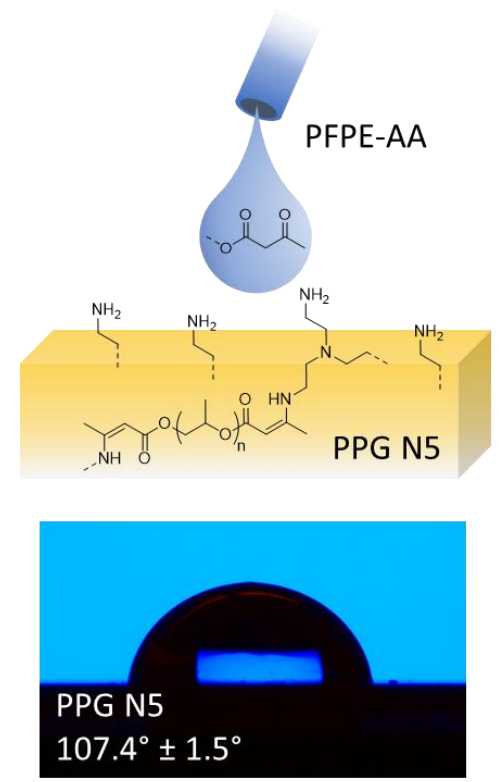
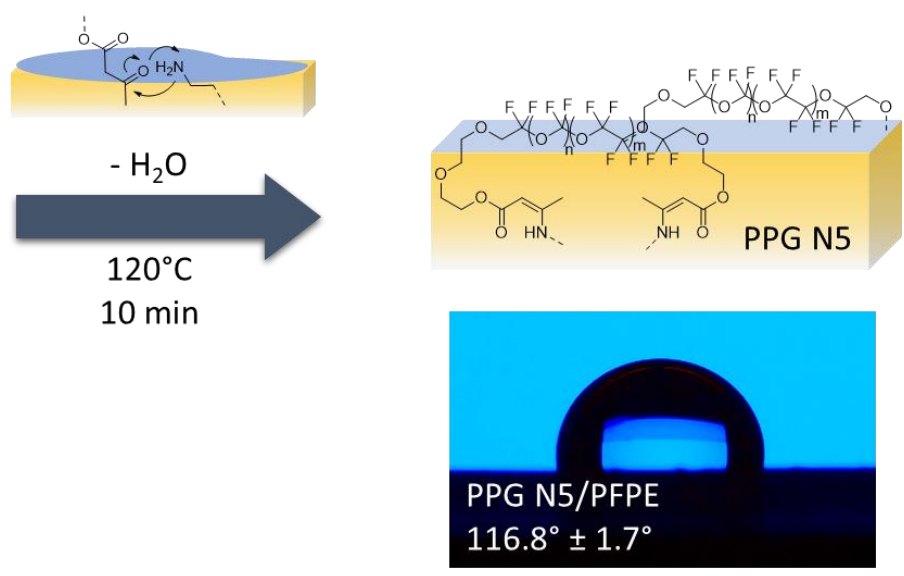


\section{WILEY-VCH}

Figure 6. Schematic display of the coating of a non-fluorinated PPG-based vinylogous urethane vitrimer with a PFPE-AA monomer and the visualized change in surface properties based on the material's change in terms of water contact angle.

The difference, also in comparison to the contact angle of bulk PFPE N5, was related to quenching available primary amine functional groups on the surface and having the fluorinated chains mainly staying at the air/material interface, covering the vinylogous urethane parts. All of this was created, by reducing the fluorinated content to a minimum and maintaining the bulk reshape-ability properties of the respective material. In other words, it did not create a macroscopically separated interface, which would result in turbidity. This experiment demonstrates tremendous degrees of flexibility in the choice of the bulk material properties, also allowing for example potentially increased mechanical robustness by choosing a higher $T_{g}$ material as a coated substrate. Furthermore, this approach indicates that lamination of different CANs, possessing compatible functional groups is a direct approach to achieve desired surface functionalities while maintaining strong adhesive forces, even if the combined materials are seemingly incompatible.

\section{Conclusion}

In conclusion, it was demonstrated that dynamic permanent networks based on transamination of vinylogous urethanes provide a versatile material platform for physical structuration and chemical post-modification. A wide range of structural dimensions, from several microns to patterns of around $300 \mathrm{~nm}$, were created. Interestingly, associated with the use of fluorinated vitrimers, those submicron patterns remained visible for up to three weeks, in contrast to non-fluorinated elastic materials. It also resulted in a noticeable change in contact angle, similar to chemical surface modification with a fluorinated monomer. Furthermore, the assembly of incompatible matrices was achieved. Strong adhesive forces were demonstrated as a peel test of laminated fluorinated and non-fluorinated films resulted in cohesive failure. The 


\section{WILEY-VCH}

presented findings thus demonstrate the versatility of vinylogous urethane vitrimers for assembly, structuration and chemical post-modification of permanently crosslinked networks. This approach enables almost endless possibilities for the creation, assembly or postmodification of permanently crosslinked polymer networks. With this methodology, specifically targeted function or surface functionality is achievable, such as facile, yet strong adhesion of fluorinated materials. Moreover, (re)processing and rapid fabrication of networks is possible for applications in e.g. micro-fluidics or lab-on-a-chip devices, soft-lithography, anti-(bio)fouling and more.

\section{Experimental Section}

Materials: Polypropylene glycol (PPG $\mathrm{M}_{\mathrm{n}}=400 \mathrm{~g} \cdot \mathrm{mol}^{-1}$ ), tert-butylacetoacetate (TBAA, $>98 \%$ ), and tris(2-aminoethyl)amine (TREN, 96\%), and polycaprolactone-blockpolytetrahydrofuran-block-polycaprolactone $\left(\mathrm{M}_{\mathrm{n}}=2000 \mathrm{~g} \cdot \mathrm{mol}^{-1}\right)$ were purchased from SigmaAldrich and used without further purification. Fluorolink E10H (PFPE-OH, $\mathrm{M}_{\mathrm{n}} \approx 2000 \mathrm{~g} \cdot \mathrm{mol}^{-1}$ ) was purchased from Acota (UK). Para-toluene sulfonic acid monohydrate ( $\left.p \mathrm{TsOH}^{*} \mathrm{H}_{2} 0,98 \%\right)$ was purchased from TCI, polyisocyanate resin of hexamethylene diisocyanate (Desmodur N3900) was donated by Covestro.

Instrumentation: Attenuated total reflection- Fourier transform infrared (ATR-FTIR) spectra were measured using a Perkin-Elmer Spectrum1000 FTIR infrared spectrometer with a diamond ATR probe. Thermogravimetric analyses (TGA) were performed with a Mettler Toledo TGA/SDTA851e instrument under nitrogen atmosphere at a heating rate of $10 \mathrm{~K} \cdot \mathrm{min}^{-1}$ from $25{ }^{\circ} \mathrm{C}$ to $800{ }^{\circ} \mathrm{C}$ to measure determine weight loss with a temperature ramp.

Differential scanning calorimetry (DSC) analyses were performed with a Mettler Toledo instrument 1/700 under nitrogen atmosphere at a heating rate of $10 \mathrm{~K} \cdot \mathrm{min}^{-1}$.

Rheology experiments were performed on an Anton Paar MCR 302. The experiments were performed in parallel plate geometry using $8 \mathrm{~mm}$ sample disks. Unless otherwise specified, the 


\section{WILEY-VCH}

experiments were performed using a normal force of $1 \mathrm{~N}$, an oscillating frequency of $1 \mathrm{rad} . \mathrm{s}^{-1}$ and a strain of $1 \%$. For each rheology experiment, the applied stress was within the linear viscoelastic region at the measured temperatures. Time sweep experiments were performed at a constant temperature and G' and G', were measured as a function of time. For stress relaxation experiments, a strain of $1 \%$ was applied to the material and the relaxation modulus $\left(\mathrm{G}^{*}(\mathrm{t})\right)$ was followed over time at a constant temperature.

Atomic force microscopy (AFM) was performed using a Dimension 3100 AFM (Veeco Instruments, Plainview, USA) for the silicon molds and a Nanosurf easyScan2 Atomic Force Microscope (Nanosurf, Switzerland) using a $110 \mu \mathrm{m}$ scan head for the interface of the laminates.

Scanning electron microscopy (SEM; Hitachi SU3500) and energy dispersive spectroscopy (EDS; Oxford INCA, Bruker Quantax) by EAG laboratories was performed to characterize the interfaces of vitrimer laminates. Field emission SEM (JEOL JSM-7401F, JEOL, Japan) was used to characterize surface patterns of polymer networks as well as optical microscopy (Nikon Eclipse Ci) of Sharklet ${ }^{\circledR}$-patterned films.

Contact angle analyses (Ossilla, Ossila Ltd, UK; using a polynomial fit) was conducted by recording a video of a drop falling on the surface and determining the contact angle when a first stable shape was reached. Five repetitions per sample were performed, averaged, and were reported including the standard deviation.

Monomer synthesis: The acetoacetylated monomers were obtained from an earlier reported synthetic procedure, following a vacuum distillation starting from the fluorinated and nonfluorinated polyether diols (PFPE-OH, PPG-OH respectively). ${ }^{[4 b, 21 d]}$ Therefore, 1 eq. of the starting diol was combined with 2.1 eq. of TBAA, heated to $130^{\circ} \mathrm{C}$ and mixed for $1 \mathrm{~h}$. Formed tert-butanol was continuously removed until reaction came to completion, as indicated by a drop of the still head temperature to $40^{\circ} \mathrm{C}$. Residual TBAA was removed via distillation at $130^{\circ} \mathrm{C}$ below 2 mbar, and the formation of the final product was confirmed via NMR. 


\section{WILEY-VCH}

Network synthesis: Vinylogous urethane networks with 5 or 20 mol.\% excess of primary amines (compared to acetoacetate moieties, labelled as N5, or N20 respectively) with and without para-toluene sulfonic acid $(p \mathrm{TsOH})$ as a catalyst were created as follows. For polymer networks containing $p$ TsOH, first 0.06 eq. (PPG N5H6, PPG N20H6, PFPE N5H6, PFPE $\mathrm{N} 20 \mathrm{H} 6$ ) of catalyst were dissolved in 0.35 eq. ( 5 mol.\% excess amines) or 0.4 eq. (20 mol. \% excess amines) of tris(2-aminoethyl)amine (TREN) upon mixing in a polypropylene cup until a clear solution was obtained, to which the acetoacetylated monomer (PFPE-AA, or PPG-AA) was added, followed by mixing for $2 \mathrm{~min}$ at $2500 \mathrm{rpm}$ using a DAC $150.1 \mathrm{FVZ}$ speed mixer. Polymer networks without a catalyst were created by combining 0.5 eq. polyether bisacetoacetate and 0.35 eq. ( 5 mol.\% excess) of TREN in a polypropylene cup, and subsequently mixing for $2 \mathrm{~min}$ with a speed of $2500 \mathrm{rpm}$. Then, the cup was placed in an oven at $80{ }^{\circ} \mathrm{C}$ for up to $4 \mathrm{~h}$ to complete the network formation. Hereafter, the polypropylene cup was broken, and the network was cured and dried overnight at $70^{\circ} \mathrm{C}$ under vacuum. The dried sample was cut into pieces and ca. $10 \mathrm{~g}$ of fluorinated vitrimer were used to fill a rectangular steel mold (70 mm x $40 \mathrm{~mm} \times 2 \mathrm{~mm})$, or ca. $2 \mathrm{~g}$ for a smaller mold (30 $\mathrm{mm} \times 15 \mathrm{~mm} \times 2 \mathrm{~mm})$ and reprocess the material by applying 2 tons of pressure at $150^{\circ} \mathrm{C}$ to a pre-heated mold for $5-10$ min (catalyzed) or 30 min (non-catalyzed). A permanently crosslinked polyurethane network was prepared by mixing $1.7 \mathrm{~g}$ PCL-block-pTHF-block-PCL and $0.3 \mathrm{~g}$ of a multifunctional isocyanate (Desmodur N3900) with a ratio of $\mathrm{OH} / \mathrm{NCO}$ of $1: 1$ at $50^{\circ} \mathrm{C}$ until a homogenous mixture was obtained. The viscous mixture was degassed under vacuum, brought between two glass slides with a $250 \mu \mathrm{m}$ height spacer and cured at $70^{\circ} \mathrm{C}$ in an oven overnight. Lamination of plates was performed by placing a PFPE N5 plate on top of a PPG N5 plate and compressing them at $150^{\circ} \mathrm{C}$ under 2 tons of pressure for $20 \mathrm{~min}$. For the interface analysis, a thin stripe of the laminated material was cut with a razorblade and placed between glass-slides (mentioned glass-slides are typically Rainex $(\mathbb{C}$ coated), which were held together with paper clips and the set-up was placed in an oven at $120^{\circ} \mathrm{C}$ for 60 min to create a smoother surface for SEM and 


\section{WILEY-VCH}

AFM analyses. For the formation of thin vitrimer films, a small piece of the vitrimer sheet was positioned between two glass slides and held together with paper clips and placed in an oven at $120^{\circ} \mathrm{C}$ to reshape the materials into films of 200 to $600 \mu \mathrm{m}$ thickness suitable for nanoimprinting and surface analyses. Surface coating using PFPE-AA monomer was performed by applying a drop of this compound with a $200 \mu \mathrm{l}$ micro-pipette onto a vitrimer film, placed between two glass-slides and put in the oven at $120^{\circ} \mathrm{C}$ for $10 \mathrm{~min}$. Any residual monomer was removed with an acetone containing paper cloth.

Nano-imprint lithography: Thermally activated nano-imprint lithography was performed on an Eitre 3 (Obducat, Lund, Sweden), using different patterned silicon molds (pillar, hole, line patterned) without the use of a non-adhesive silane. A polymer network film was placed on a silicon wafer on top of which a silicon mold was put. The set-up was positioned in a chamber, covered with a PET-film and closed. The chamber was heated to $130^{\circ} \mathrm{C}$ and imprinting was performed at 25 bar air-pressure for a duration of $150 \mathrm{~s}(\mathrm{~N} 20 \mathrm{H} 6), 300 \mathrm{~s}(\mathrm{~N} 5 \mathrm{H} 6)$ and $900 \mathrm{~s}$ (non-catalyzed and permanently crosslinked reference), which was followed by a gradual cooling step under similar pressure. Afterwards the set-up was removed, and the imprinted sample carefully separated and peeled-off from the silicon mold. The silicon-mold was cleaned by placing it in acetone and carefully removing swollen polymer residues with a paper cloth and compressed air.

\section{Supporting Information}

Supporting Information is available from the Wiley Online Library or from the author.

\section{Acknowledgements}

C.T. and M.G. acknowledge the Research Foundation-Flanders (FWO) for Ph.D. and Postdoctoral fellowships. F.E.D.P. thanks BOF-UGent (GOA-funding). This research was supported in part by the Colorado Shared Instrumentation in Nanofabrication and Characterization (COSINC): the COSINC-CHR (Characterization), College of Engineering \& 


\section{WILEY-VCH}

Applied Science, University of Colorado Boulder. Prof. Yifu Ding, Dr. Maciej Podgorski, Dr.

Matthew Barros, Dr. Tomoko Borsa, Adam Dobson, Alina Martinez, Bernhard de Meyer and

Shouhong Fan are gratefully acknowledged for technical support and use of equipment. Prof.

Johan M. Winne and Dr. Nezha Badi are thanked for fruitful discussions.

\section{Conflict of Interest}

The authors declare no conflict of interest.

Received: ((will be filled in by the editorial staff))

Revised: ((will be filled in by the editorial staff))

Published online: ((will be filled in by the editorial staff))

\section{References}

[1] L. Imbernon, S. Norvez, Eur. Polym. J. 2016, 82, 347.

[2] a) L. M. Cox, A. M. Martinez, A. K. Blevins, N. Sowan, Y. Ding, C. N. Bowman, Nano Today 2020, 31, 100838; b) H. N. Hansen, R. J. Hocken, G. Tosello, CIRP Annals 2011, 60, 695.

[3] a) C. N. Bowman, C. J. Kloxin, Angew. Chem. Int. Ed. 2012, 51, 4272; b) M. Podgorski, B. D. Fairbanks, B. E. Kirkpatrick, M. McBride, A. Martinez, A. Dobson, N. J. Bongiardina, C. N. Bowman, Adv. Mater. 2020, 32, e1906876.

[4] a) M. Röttger, T. Domenech, R. van der Weegen, A. Breuillac, R. Nicolaÿ, L. Leibler, Science 2017, 356, 62; b) C. Taplan, M. Guerre, J. M. Winne, F. E. Du Prez, Mater. Horiz. 2020, 7, 104; c) B. Zhang, K. Kowsari, A. Serjouei, M. L. Dunn, Q. Ge, Nat. Commun. 2018, 9, 1831; d) D. T. Sheppard, K. Jin, L. S. Hamachi, W. Dean, D. J. Fortman, C. J. Ellison, W. R. Dichtel, ACS Cent Sci 2020, 6, 921.

[5] a) Y. Liu, Z. Tang, Y. Chen, C. Zhang, B. Guo, ACS Appl. Mater. Interfaces 2018, 10, 2992; b) Y. Spiesschaert, M. Guerre, L. Imbernon, J. M. Winne, F. Du Prez, Polymer 2019, 172, 239; c) T. Philip, N. Huagang, Z. Chengpu, Y. Kai, L. Samuel, J. Yinghua, Q. H. Jerry, Z. Wei, Adv. Mater. 2016, 28, 2904.

[6] a) Y. Yang, Z. Pei, X. Zhang, L. Tao, Y. Wei, Y. Ji, Chem. Sci. 2014, 5, 3486; b) M. Capelot, D. Montarnal, F. Tournilhac, L. Leibler, J. Am. Chem. Soc. 2012, 134, 7664.

[7] a) Y. Ito, D. Aoki, H. Otsuka, Polym. Chem. 2020, 11, 5356; b) A. L. Dobson, N. J. Bongiardina, C. N. Bowman, ACS Applied Polymer Materials 2020, 2, 1053.

[8] a) Z. Pei, Y. Yang, Q. Chen, E. M. Terentjev, Y. Wei, Y. Ji, Nature Materials 2014, 13, 36; b) M. K. McBride, A. M. Martinez, L. Cox, M. Alim, K. Childress, M. Beiswinger, M. Podgorski, B. T. Worrell, J. Killgore, C. N. Bowman, Science Advances 2018, 4, eaat4634.

[9] D. Montarnal, M. Capelot, F. Tournilhac, L. Leibler, Science 2011, 334, 965.

[10] a) W. Denissen, J. M. Winne, F. E. Du Prez, Chem. Sci. 2016, 7, 30; b) M. Guerre, C. Taplan, J. M. Winne, F. E. Du Prez, Chem. Sci. 2020, 11, 4855.

[11] J. M. Winne, L. Leibler, F. E. Du Prez, Polym. Chem. 2019, 10, 6091.

[12] M. Capelot, M. M. Unterlass, F. Tournilhac, L. Leibler, ACS Macro Lett. 2012, 1, 789. 


\section{WILEY-VCH}

[13] Y. Nishimura, J. Chung, H. Muradyan, Z. Guan, J. Am. Chem. Soc. 2017, 139, 14881.

[14] Z. Q. Lei, H. P. Xiang, Y. J. Yuan, M. Z. Rong, M. Q. Zhang, Chem. Mater. 2014, 26, 2038.

[15] C. Wang, T. M. Goldman, B. T. Worrell, M. K. McBride, M. D. Alim, C. N. Bowman, Mater. Horiz. 2018, 5, 1042.

[16] a) M. M. Obadia, B. P. Mudraboyina, A. Serghei, D. Montarnal, E. Drockenmuller, J. Am. Chem. Soc. 2015, 137, 6078; b) B. Hendriks, J. Waelkens, J. M. Winne, F. E. Du Prez, ACS Macro Lett. 2017, 6, 930.

[17] a) W. Denissen, I. De Baere, W. Van Paepegem, L. Leibler, J. Winne, F. E. Du Prez, Macromolecules 2018, 51, 2054; b) W. Denissen, G. Rivero, R. Nicolaÿ, L. Leibler, J. M. Winne, F. E. Du Prez, Adv. Funct. Mater. 2015, 25, 2451.

[18] a) R. L. Snyder, D. J. Fortman, G. X. De Hoe, M. A. Hillmyer, W. R. Dichtel, Macromolecules 2018, 51, 389; b) D. J. Fortman, J. P. Brutman, C. J. Cramer, M. A. Hillmyer, W. R. Dichtel, J. Am. Chem. Soc. 2015, 137, 14019; c) C. He, S. Shi, D. Wang, B. A. Helms, T. P. Russell, J. Am. Chem. Soc. 2019, 141, 13753; d) P. Taynton, K. Yu, R. K. Shoemaker, Y. Jin, H. J. Qi, W. Zhang, Adv. Mater. 2014, 26, 3938; e) J. S. A. Ishibashi, J. A. Kalow, ACS Macro Lett. 2018, 7, 482.

[19] a) G. B. Lyon, L. M. Cox, J. T. Goodrich, A. D. Baranek, Y. Ding, C. N. Bowman, Macromolecules 2016, 49, 8905; b) F. Lossada, J. Guo, D. Jiao, S. Groeer, E. Bourgeat-Lami, D. Montarnal, A. Walther, Biomacromolecules 2019, 20, 1045; c) L. Imbernon, S. Norvez, L. Leibler, Macromolecules 2016, 49, 2172; d) Z. Chen, Q. Shi, X. Kuang, H. J. Qi, T. Wang, Composites Part B: Engineering 2020, 194, 108054; e) C. He, S. Shi, X. Wu, T. P. Russell, D. Wang, J. Am. Chem. Soc. 2018, 140, 6793.

[20] W. Denissen, M. Droesbeke, R. Nicolay, L. Leibler, J. M. Winne, F. E. Du Prez, Nat. Commun. 2017, 8, 14857.

[21] a) Y. Spiesschaert, C. Taplan, L. Stricker, M. Guerre, J. M. Winne, F. E. Du Prez, Polym. Chem. 2020, 11, 5377; b) Y. Spiesschaert, M. Guerre, I. De Baere, W. Van Paepegem, J. M. Winne, F. E. Du Prez, Macromolecules 2020, 53, 2485; c) L. Bai, J. Zheng, Compos. Sci. Technol. 2020, 190, 108062; d) M. Guerre, C. Taplan, R. Nicolaÿ, J. M. Winne, F. E. Du Prez, J. Am. Chem. Soc. 2018, 140, 13272; e) J. J. Lessard, G. M. Scheutz, R. W. Hughes, B. S. Sumerlin, ACS Applied Polymer Materials 2020, 2, 3044.

[22] B. T. Worrell, M. K. McBride, G. B. Lyon, L. M. Cox, C. Wang, S. Mavila, C. H. Lim, H. M. Coley, C. B. Musgrave, Y. Ding, C. N. Bowman, Nature communications 2018, 9, 2804. 


\section{WILEY-VCH}

This study shows that vinylogous urethane chemistry is a versatile platform for both physical post-modification on micro and nanoscale, and chemical post-modification of permanent, yet dynamic covalent polymer networks.

Christian Taplan, Marc Guerre, Christopher N. Bowman*, and Filip E. Du Prez*

Surface modification of (non)-fluorinated vitrimers through dynamic transamination ToC figure

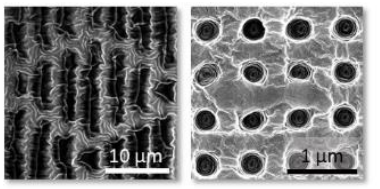

Alter your surface with

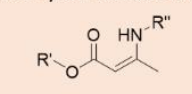

vinylogous urethane

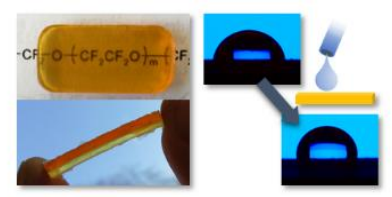

